\title{
Reconfigurable Modular Antenna for Near-Field UHF RFID Smart Point Readers
}

\author{
Andrea Michel, Member, IEEE, Marcos Rodriguez Pino, and Paolo Nepa, Member, IEEE
}

\begin{abstract}
In this paper, a reconfigurable modular antenna is presented for smart point readers employed in near-field RFID applications at the UHF ETSI band $(865-868 \mathrm{MHz})$. The antenna comprises two modules sharing the same $250 \times 250 \mathrm{~mm}^{2}$ aperture: a spiral-shaped travelling wave antenna and an array of four resonating slot antennas connected in series to the travelling wave antenna through a printed matching/delay network. The electromagnetic field distribution generated by the overall antenna can be controlled and shaped through an absorptive RF switch placed at the end of the spiral travelling wave antenna. Specifically, two configurations can be selected, on the basis of the particular operating scenario. In the Spiral TWA Configuration, that is the best for tag writing operations, the electromagnetic field is maximized in the reader central area, right on its surface only (reactive near-field region). In the Modular Antenna Configuration, a field coverage is guaranteed up to a few decimeters away from the reader surface (radiative near-field region). Measurements on a prototype and simulation results are in a good agreement. Moreover, a system-level characterization has been carried out and compared to a numerical analysis aimed to qualitatively predict tag detection performance.
\end{abstract}

Index Terms - RFID, near-field antenna, reconfigurable antenna, aperture-shared antenna, smart point reader, desktop reader.

\section{INTRODUCTION}

$\mathrm{T}$ HE increasing number of applications and scenarios in which Radio Frequency Identification (RFID) systems are employed has required researchers to design $a d-h o c$ system solutions, especially for Ultra-High Frequency (UHF) RFID systems in pharmaceutical and retail industries. Licensed UHF RFID operating frequency bands are allocated within the 860 to $960 \mathrm{MHz}$ band, depending on the geographical region: as for example, ETSI band (865-868 MHz) in Europe, FCC band (902-928 MHz) in North and South America, and 952 - 957.6 in Japan (effective until March 31, 2018) [1]. UHF RFID systems allow for a high read- and data-rate and for small size tags. However, the item material the tag is attached to and the mutual coupling among tags in a stacked configuration can compromise the UHF RFID system performance, mainly affecting the tag readability and reducing the read range. Thus, Near-Field (NF) UHF RFID systems have been recently introduced to maximize and confine the electric and magnetic fields within a limited volume around the reader [1]-[4]. Also, the far-field radiation efficiency is quite low, so reducing the detection of tags that are placed far from the reader (false positive issues).

In the last decade, various antennas have been proposed for NF UHF RFID readers [5]- [32]. The most common NF UHF RFID radiating elements are the loop-like resonant antennas [5]-[12]. In particular, Segmented loops have been introduced to reduce the electrical size of a loop antenna while maintaining the same physical size [5]-[10], generating a strong and uniform magnetic field distribution in proximity of the antenna surface. Furthermore, Transmission Line (TL) antennas [13]-[19] are proposed for NF UHF RFID systems, typically based on microstrip [13]-[15], Coplanar Stripline (CPS) [16] or Coplanar Waveguide (CPW) [17]-[19] technologies. Generally, thanks to the presence of a matched termination, the leaky transmission line antennas exhibit limited far-field radiation and large impedance bandwidth, making the input impedance matching less sensitive to the presence of the tagged items in the antenna near-field region. Resonant antennas and arrays have also been proposed [20]-[26]. Since their size is strictly related to the operating frequency, miniaturization techniques are typically used to limit the antenna size at UHF band, so allowing the antenna to be integrated in reader cases. A more detailed state-of-the-art analysis on antennas for NF UHF RFID applications is described in [26].

From a practical point of view, all these antennas can detect tags up to few tens of centimeters when only few tags are considered. More critical scenarios are represented by tags applied to objects with different materials or multiple tags, where a higher field intensity is required to detect each tag. Thus, reader antennas that can exhibit in unloaded conditions a read range larger than that required in operational conditions have been recently proposed [28]-[32]. In this framework, Reconfigurability is becoming an interesting antenna feature, which can make the NF UHF RFID system more adaptable to the specific application scenario. It would allow for a shaping of the interrogation field in the antenna near-field region, when a simple control of the reader output power is not enough to guarantee high successful reading percentages on the whole antenna surface, for any tagged item and tag topology/orientation and for different operating conditions. As an example, an antenna can be reconfigured to extend or reduce the detection volume size on the basis of the specific operating scenario, so compensating the field attenuation due to the presence of items made of different materials. In [33], a reconfigurable passive UHF RFID ETSI (865-868 MHz) loop 
reader antenna for near-field and far-field RFID applications is presented. The radiation properties of the antenna can be reconfigured by employing varactor diodes. In [34] the operating frequency and radiation properties of a printed loop antenna are modified by using varactor diodes. Thus, the antenna is capable of changing the radiation pattern (i.e. omnidirectional or broadside) while guaranteeing a relatively strong and uniform magnetic field at the center of the loop. Furthermore, since RFID tag detection at UHF band is due to both inductive and capacitive coupling, the electric and magnetic field distributions within a confined volume can be adjusted by reconfiguring the radiating element layout by means of varactors or RF switches. Also, reconfigurability can be suitable to maximize a single field component, so that the relative orientation of the tag could be estimated. Thus, above cited reconfigurable system may be able to detect the loop-like tags positioned with different orientation.

In this paper, a Reconfigurable Modular Antenna is proposed for near-field UHF RFID applications. Specifically, a travelling wave antenna is combined with resonating antennas, which share the surface of the desktop reader antenna. When the TWA antenna ends on a matched load (Spiral TWA Configuration), strong and uniform electric and magnetic fields up to a few centimeters from the antenna surface (near-field reactive region) are generated. Such a configuration is suitable especially for writing operations, where a higher field intensity is required. In the proposed layout, the TWA is represented by a spiral microstrip line in order to distribute the electromagnetic (EM) energy among all the field components, which is important to guarantee the detection of tags arbitrarily oriented with respect to the reader antenna. Alternatively, the spiral microstrip line can feed a resonating antenna or an array of resonating antennas (Modular Antenna Configuration) [28]- [32], so covering the radiative near-field region up to a few tens of centimeters from the antenna surface, yet radiating a relatively low field in the antenna far-field region as required by antennas for desktop readers. In this way, it is possible to improve the tag detection up to few decimeters from the antenna surface, even in presence of stacks of tags. In the proposed layout, the resonating antenna element is represented by an array of four curved slot antennas which share the TWA aperture (aperture-shared antenna configuration). The slots are 90-degree-rotated with respect to the antenna center, and they are fed by the microstrip transmission line through a matching/delay network. Such a network is responsible for feeding each radiating element with currents exhibiting the same amplitude but with a 90-degree phase difference, so implementing the sequential rotation feeding technique and achieving a circularly polarized radiated field. Then, an absorptive RF switch is added to the end of the spiral microstrip line, and it is used to enable the proper antenna operating mode on the basis of the specific scenario. It is worth noting that by activating the proper radiating element, the field distribution generated by the Reconfigurable Modular Antenna changes, giving different system performance without increasing the reader output power level. In Section II, the antenna layout and the simulated and measured performance are presented and discussed, in terms of input impedance matching, electric field distribution, radiation pattern, gain and axial ratio. A system-level antenna performance prediction is also proposed in Section III, and compared with the measured Received Signal Strength Indicator (RSSI). The antenna has been designed by using the CST Microwave Studio ${ }^{\circledR}$ software.

\section{ANTENNA Layout AND PERFORMANCE}

The proposed antenna $\left(250 \times 250 \mathrm{~mm}^{2}\right)$ has been designed to be easily integrated in a smart point reader or desktop reader for near-field applications in the UHF RFID ETS band (865-868 MHz). The overall antenna consists of 3 main parts: an internal $50 \Omega$ spiral-shaped microstrip line, a printed matching/delay network and an array of four resonating curved slots etched on the antenna ground plane (Fig. 1). The antenna is printed on a grounded $1.53 \mathrm{~mm}$-thick FR4 $(\varepsilon r=4.4, \operatorname{tg} \delta=0.025)$ dielectric substrate. The main antenna dimensions are listed in Table I.

The spiral geometry of the internal microstrip line has been chosen in order to distribute the electromagnetic (EM) energy among all the field components, which allows for a more reliable detection of tags laying on the antenna surface, that is with a minimal dependence on the tag position and orientation. In the Spiral TWA Configuration, the microstrip line ends on a matched load, while in the Modular Antenna Configuration it is connected to the matching/delay network, and then it feeds the resonating curved slots. In both configurations, a current standing wave is avoided, so guaranteeing a field uniformity above the reader surface. Moreover, thanks to the presence of the TWA, return loss requirement can be met in a relatively large bandwidth. A distance between the spiral lines equal to $10 \mathrm{~mm}$ has been chosen in order to minimize the coupling effects between them and therefore the characteristic impedance variations. It is worth noting that a longer line can fit a larger area. 


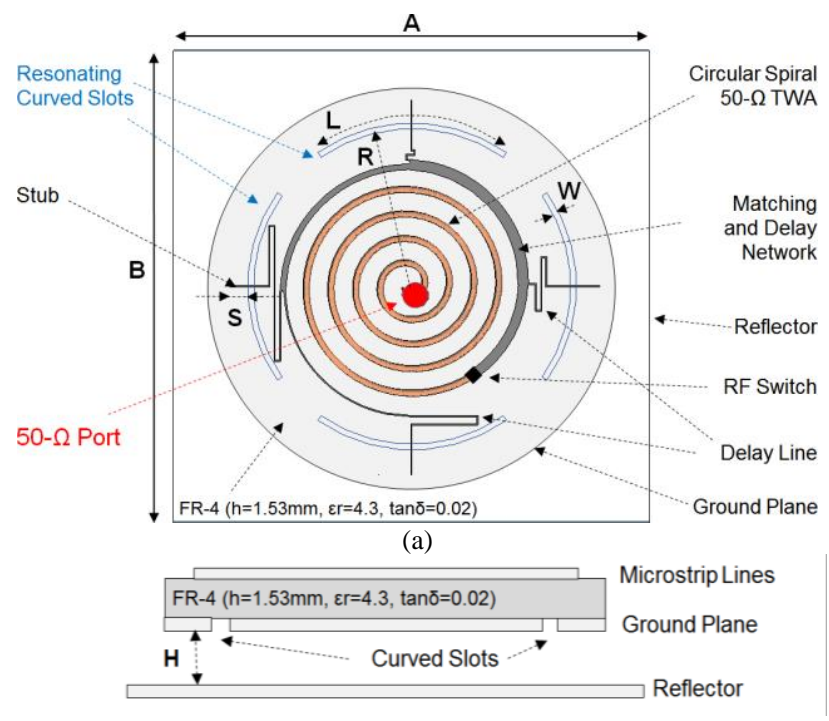

(b)

Fig. 1 - Antenna (a) top and (b) lateral view.

TABLE I

ANTENNA MAIN PARAMETERS

\begin{tabular}{|c|c|c|c|}
\hline \multicolumn{2}{|c|}{ GEOMETRICAL PARAMETERS (Fig. 1) } & \multicolumn{2}{|c|}{ TWA IMPEDANCES AND LENGTHS (Fig. 2) } \\
\hline Parameter & Value & Parameter & Value \\
\hline $\mathbf{A}$ & $250 \mathrm{~mm}$ & $\mathbf{R}_{\mathrm{DLi}}, \mathbf{Z}_{\mathrm{ANT}}$ & $100 \Omega$ \\
\hline B & $250 \mathrm{~mm}$ & $\mathbf{R}_{\mathrm{L} 2}$ & $50 \Omega$ \\
\hline $\mathbf{H}$ & $40 \mathrm{~mm}$ & $\mathbf{R}_{\mathrm{L} 1}$ & $33.33 \Omega$ \\
\hline $\mathbf{S}$ & $6 \mathrm{~mm}$ & $\mathbf{R}_{\mathrm{TR}}$ & $35.35 \Omega$ \\
\hline $\mathbf{W}$ & $3 \mathrm{~mm}$ & $\mathbf{R}_{\mathbf{0}}$ & $50 \Omega$ \\
\hline $\mathbf{L}$ & $128 \mathrm{~mm}$ & $\ell_{1}$ & $82.2 \mathrm{~mm}$ \\
\hline \multirow[t]{3}{*}{$\mathbf{R}$} & $85 \mathrm{~mm}$ & $\ell_{2}$ & $26.6 \mathrm{~mm}$ \\
\hline & & $\ell_{3}$ & $156.7 \mathrm{~mm}$ \\
\hline & & $\ell_{4}$ & $192.5 \mathrm{~mm}$ \\
\hline
\end{tabular}

When the Modular Antenna Configuration is enabled, the spiral TWA is connected to the matching/delay network, which supplies in-series the four slots with currents characterized by almost the same amplitude and 90-degree-phase difference to achieve circular polarization [35]. To optimize such a network, the layout shown in Fig. 2a has been considered. In particular, each delay line has been terminated on a matched load before the resonating slots. Then, four ports (Port 2 - Port 5) have been introduced to compute the $S_{i, 1}$ parameters and estimate the currents amplitude and phase-shift. An equivalent circuit of the entire antenna is shown in Fig. 2b. Each resonating slot antenna is fed by a $100 \Omega$ microstrip line. The internal $50 \Omega$ spiral TWA is directly connected to a $\lambda_{\mathrm{g}} / 4$ transformer, which allows the impedance matching to an impedance of $25 \Omega\left(\mathrm{Z}_{\mathrm{V} ; \mathrm{B}}\right.$ in Fig. $\left.2 \mathrm{~b}\right)$. The four delay lines lengths $\left(\ell_{\mathrm{i}}\right)$ are optimized to get a 90-degree-phase shift [35].

The four slots are etched on the ground plane on the bottom side of the FR-4 substrate. Their length corresponds to halfwavelength, to get a resonating aperture, and their shape is slightly curved without significantly affect the input impedance at the resonant frequency. On the other hand, the curved shape is suitable to better follow the spiral line profile, allowing for a better embedding in a circular-shaped smart point reader. However, to compensate for the mutual coupling due to the proximity of the printed matching/delay network, slot actual length $(L)$ is set to $128 \mathrm{~mm}$. It is worth noting that, since the input power is partially radiated by the spiral TWA, each slot antenna is fed by a smaller power level, so guaranteeing a relatively low radiation into the far-field region and reducing the cross-readings (false positives) out of the read zone required for the NF UHF RFID reader. Furthermore, to limit the slot radiation below the antenna (i.e. toward the desktop) a $250 \times 250 \mathrm{~mm}^{2}$ metallic plate has been placed at a distance of $H=4 \mathrm{~cm}$ by means of plastic screws. The distance $H$ has been chosen as a trade-off between the antenna performance and the antenna overall thickness. Specifically, for small values of $H$ the curved slot antennas are short-circuited, resulting in a low radiation efficiency as well as an almost pure imaginary input impedance. On the other hand, it is well known that a distance of $\lambda / 4$ (i.e. $34.6 \mathrm{~cm}$ at $866 \mathrm{MHz}$ ) is required to achieve the best antenna performance. However, in this case the antenna would not be easily embeddable in commercial devices, due to its overall thickness. Thus, the chosen distance of $4 \mathrm{~cm}$ is the minimum distance that guarantees satisfactory antenna performance in terms of input impedance and radiated fields while limiting the antenna thickness. 

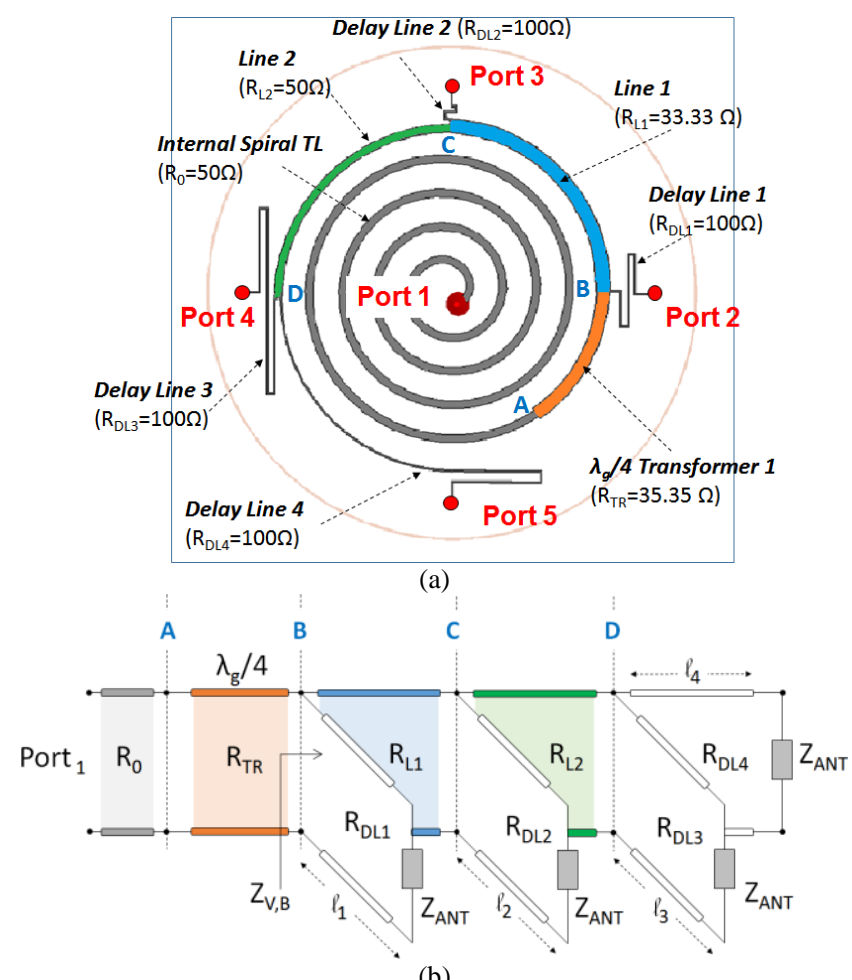

(b)

Fig. 2 - Antenna layout considered to optimize the matching and delay network (a), and its equivalent circuit (b). The four ports are placed before the resonating curved slots, which are etched on the ground plane (bottom side).

To select the operating mode, an RF switch (Analog Device ADG901 [36]) is integrated before the $\lambda_{\mathrm{g}} / 4$ transformer (section A in Fig. 2a). Thus, the transmission line can be terminated on a matched load (Spiral TWA Configuration) or it can feed the four resonating slots (Modular Antenna Configuration). In Fig. 3, the simulated current distribution on the Reconfigurable Modular Antenna is plotted at a frequency of $866 \mathrm{MHz}$, for both the operating modes. It is worth noting that for the Spiral TWA Configuration the current flows only in the internal spiral line, so concentrating an almost uniform electric field in proximity of the antenna center. On the other hand, when the Modular Antenna Configuration is activated, the current spreads out on the entire antenna surface.

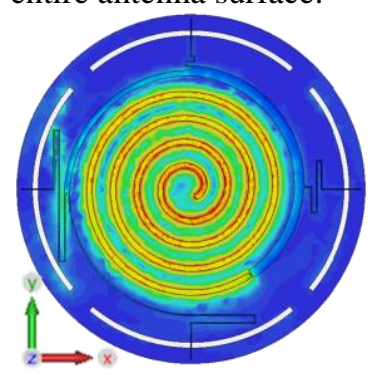

(a)
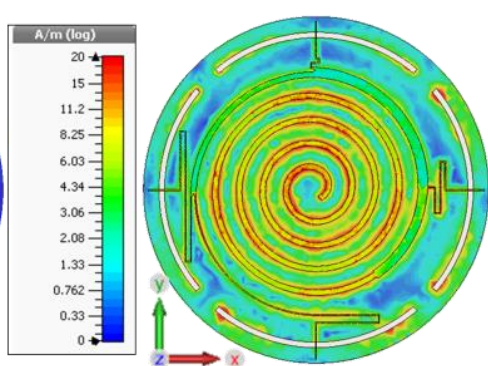

(b)

Fig. 3 - Simulated current distribution on the Reconfigurable Modular Antenna at $866 \mathrm{MHz}$, for both the (a) Spiral TWA Configuration and the (b) Modular Antenna Configuration.

A prototype has been fabricated by using the fabrication facilities available at the University of Oviedo, Spain. Some pictures are shown in Fig. 4, together with some details of the RF switch. A $3 \mathrm{~V}$ battery is used to feed the RF switch ( $\mathrm{V}_{\mathrm{DD}}$ ), and to control the switch and select the antenna configuration. The RF switch has been integrated at the bottom of the FR-4 substrate, as shown in Fig. 4c-d.

In Fig. 5 the simulated and measured reflection coefficient is shown as a function of the frequency, for both the Spiral TWA Configuration and Modular Antenna Configuration. To limit the power reflected toward the reader RF front-end, in near-field UHF-RFID applications the reflection coefficient is usually required to assume values lower than $-14 \mathrm{~dB}$. Simulated and measured results show that such a requirement is satisfied by the proposed layout in a frequency band larger than the standard UHF RFID ETSI band (865-868 MHz), for both operating modes. 


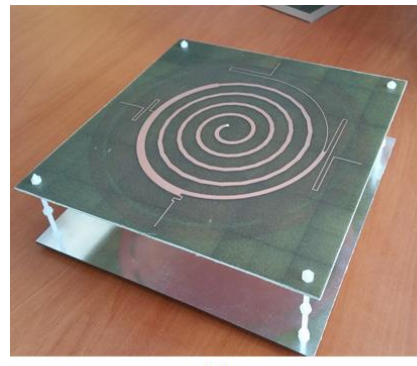

(a)

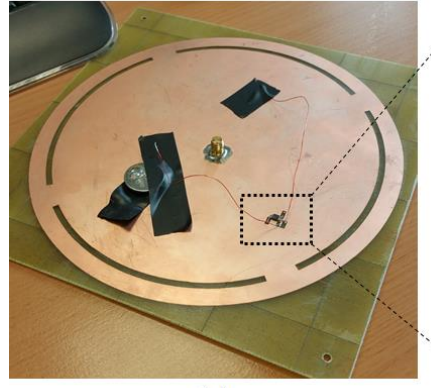

(c)

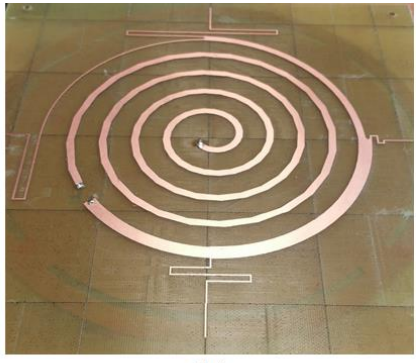

(b)

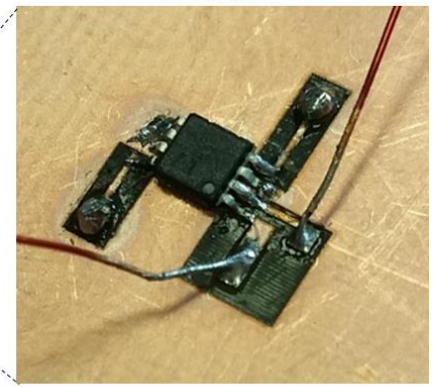

(d)

Fig. 4 - (a) Fabricated prototype and (b) top layer of the FR-4 substrate with the printed spiral TWA and matching and delay network. An absorptive RF switch has been integrated at the (c) bottom of the substrate and fed by a $3 \mathrm{~V}$ battery. A detailed picture of the RF switch is shown in (d).

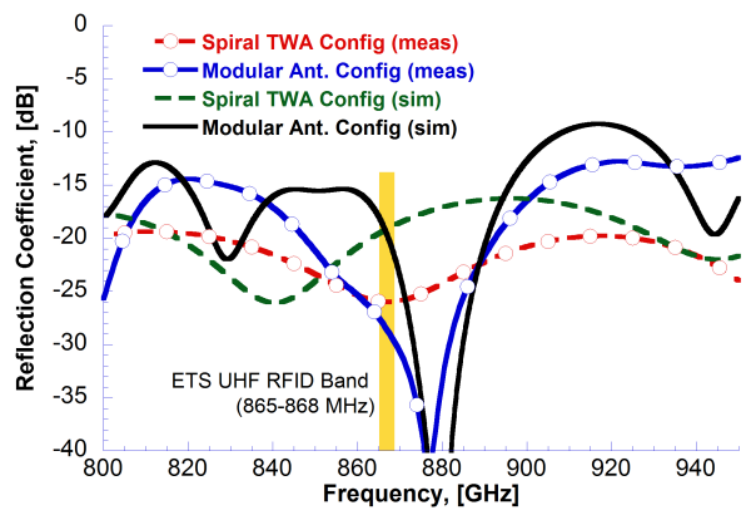

Fig. 5 - Simulated and measured reflection coefficient of both the Spiral TWA Configuration and Modular Antenna Configuration.

The performance of the Reconfigurable Modular Antennas has been measured. The measured gain is -15dB in the entire UHF RFID ETSI band, thanks to the presence of the matched load at the end of the spiral transmission line. To estimate the field uniformity close to the antenna surface, the electric field has been measured in a completely automatic near-field scanner (Fig. 6a-b). In particular, in Fig. 6a a schematic representation of the near-field system is shown and a more detailed description is presented in [37]. A small monopole has been fabricated and used as an electric near-field probe (Fig. 6c).

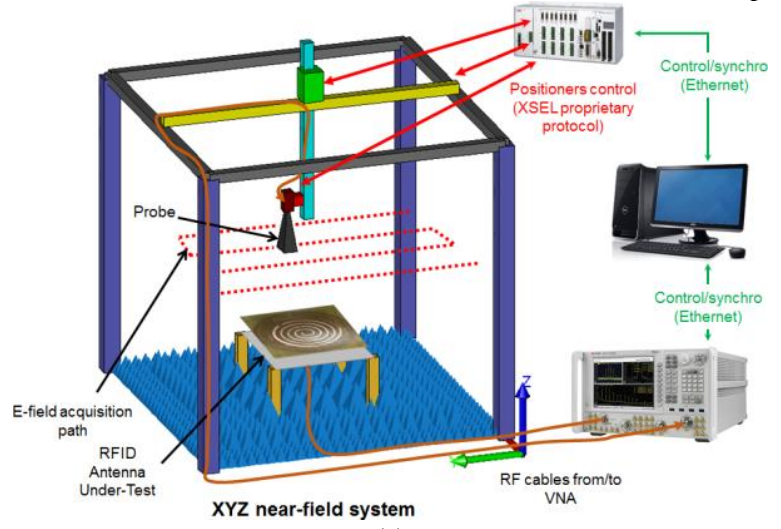

(a) 


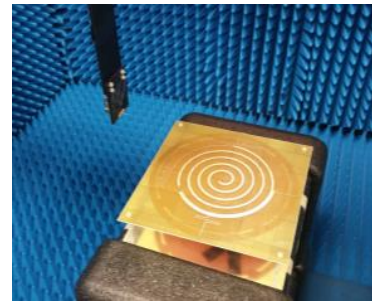

(b)

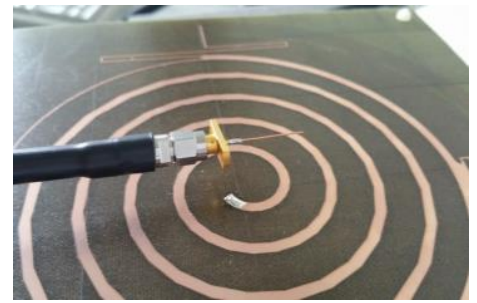

(c)

Fig. 6 - Near-field system: (a) schematic representation of the system architecture and (b) measurement setup for the near-field electric field measurements. The small electric monopole used as an electric near-field probe is shown in (c).

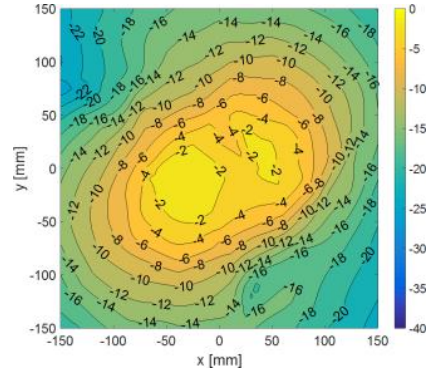

(a)

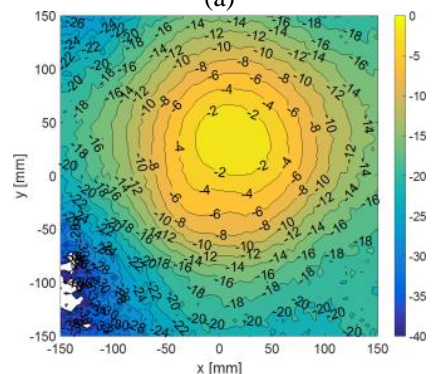

(c)

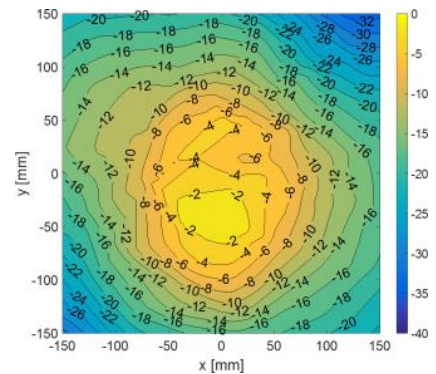

(b)

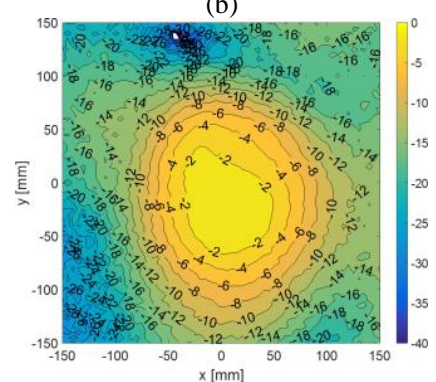

(d)

Fig. 7 - Normalized electric field at a frequency of $865 \mathrm{MHz}$, at a distance of $10 \mathrm{~cm}$ from the antenna surface, when the Spiral TWA Configuration is enabled. Simulated results: (a) X-component and (b) Y-component. Measurements: (c) X-component and (d) Y-component.

It is worth noting that such a measurement aims at evaluating the field uniformity close to the antenna rather than measuring the absolute field value. Then, the homemade electric near-field probe hasn't been calibrated before the test. Moreover, to limit the mutual coupling effect between the antenna and the mechanical arm, the electric field has been measured, starting from a distance of $10 \mathrm{~cm}$ from the antenna surface. In Fig. 7, the normalized field distribution is shown for both the tangential components ( $\mathrm{X}$ and $\mathrm{Y}$ direction in Fig. 1) and compared with the simulated normalized distribution.

The electric field normalized distributions shown in Fig. 7 demonstrate that the electric field relevant to the Spiral TWA Configuration is almost uniform and maximized in the antenna central area, for both the simulated and measured results and for both the considered tangential field components. This is desirable especially for encoding operations, because UHF RFID tags are typically placed in a target area around the antenna center, but with an arbitrary orientation with respect to the reader antenna. On the other hand, the Modular Antenna Configuration has been designed to achieve higher reading range. Hence, for such a configuration, radiated performance have been also evaluated in a far-field anechoic chamber. In Fig. 8, the axial ratio and maximum gain of the Modular Antenna Configuration are shown as a function of the frequency. The maximum measured gain is $1 \mathrm{dBic}$ at the frequency of $866 \mathrm{MHz}$, and remains almost constant in the entire UHF RFID ETSI band. The minimum measured axial ratio is $2.4 \mathrm{~dB}$ at the frequency of $858 \mathrm{MHz}$, and it is lower than $3 \mathrm{~dB}$ in the whole UHF RFID ETSI band (865-868 MHz). The simulated and measured normalized radiation pattern at the frequency of $865 \mathrm{MHz}$, for both the XZ and YZ planes, and for both the $E_{\theta}$ and $E_{\varphi}$ components, are shown in Fig. 9. It is worth noting that the simulated and measurement results are in good agreement for both the field components and cutting planes. 


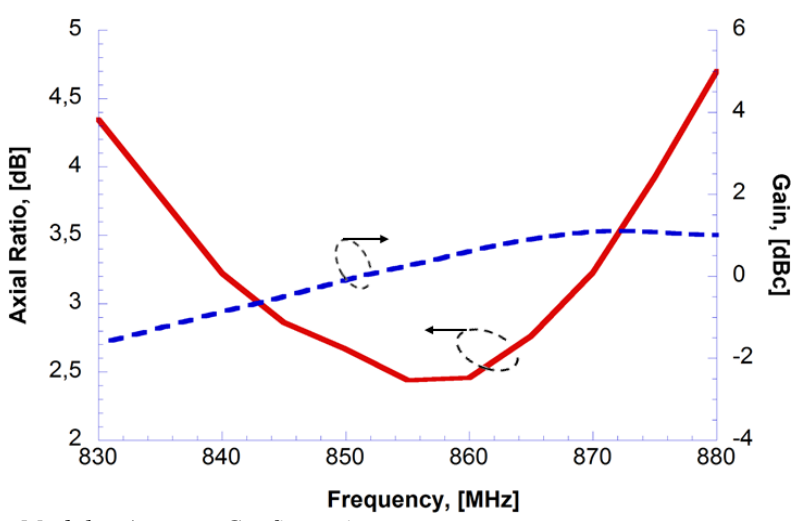

Fig. 8 - Axial ratio and maximum gain of the Modular Antenna Configuration.

Similarly to the tests carried out for the Spiral TWA Configuration, the X and Y components of the electric field at a distance of $10 \mathrm{~cm}$ from the antenna surface have been measured in the near-field chamber. Results are shown in Fig. 10 together with the normalized simulated field distribution obtained by CST Microwave Studio ${ }^{\circledR}$. A reasonable agreement between simulated and measured results has been achieved, despite some slightly differences close to the antenna borders.

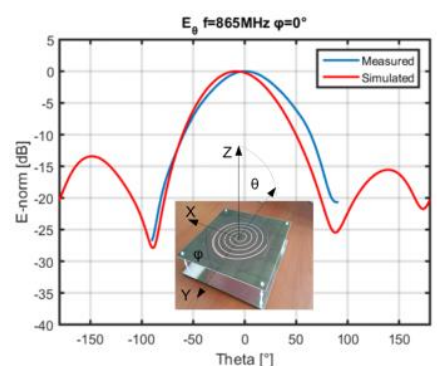

(a)

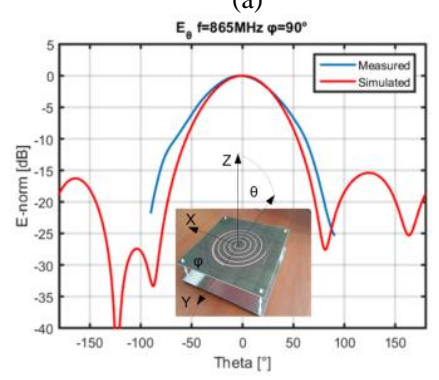

(c)

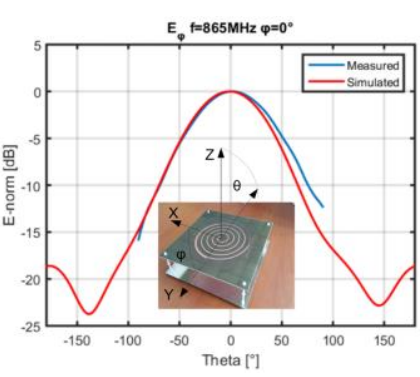

(b)

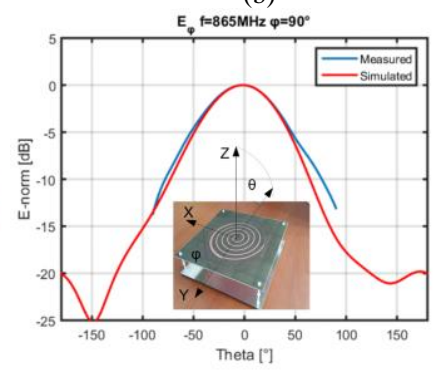

(d)

Fig. 9 - Simulated and measured radiation pattern at $865 \mathrm{MHz}$ : (a) $E_{\theta}$ and (b) $E_{\varphi}$ on the $\mathrm{XZ}$ plane $\left(\varphi=0^{\circ}\right.$ ); (c) E国 $\theta$ and (d) $E_{\varphi}$ on the YZ plane $\left(\varphi=90^{\circ}\right)$.

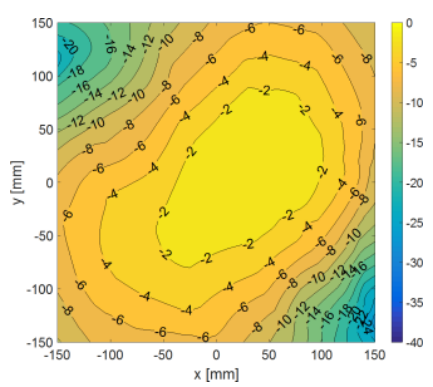

(a)

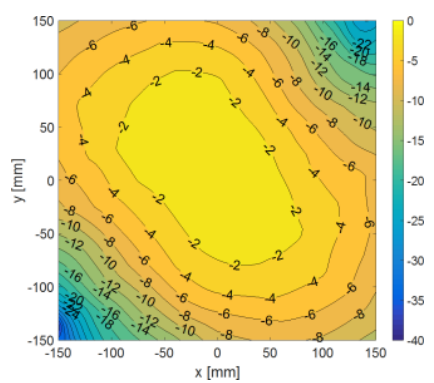

(b) 


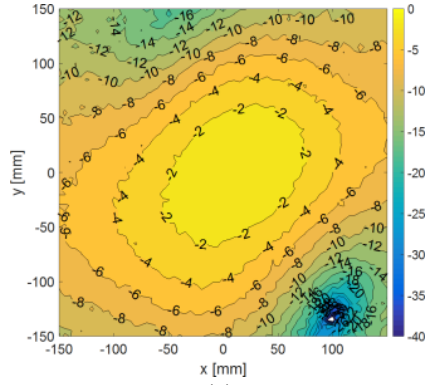

(c)

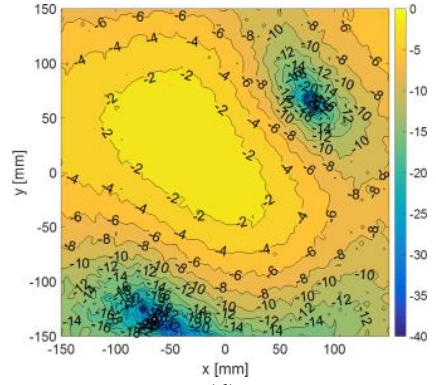

(d)

Fig. 10 - Normalized electric field at $865 \mathrm{MHz}$, at a distance of $10 \mathrm{~cm}$ from the antenna surface, when the Modular Antenna Configuration is enabled. Simulated results: (a) X-component and (b) Y-component. Measurements: (c) X-component and (d) Y-component.

\section{SYSTEM-LEVEL MEASUREMENTS}

The proposed Reconfigurable Modular Antenna has been connected to a commercial UHF RFID reader (CAEN RFID Ion, Model R4301P [38]) to carry out system level measurements in terms of reading range and tag detection. The measurement setup is shown in Fig. 11. An $8 \times 83$-cm-square cells grid has been drawn on a cardboard sheet and aligned to the antenna center, at a fixed distance from the antenna surface. Then, a LAB-ID Inlay UH100 tag [39] was placed in correspondence of each cell, with a specific orientation with respect to the radiating element. In Fig. 12, the results of the detection tests are shown for both the Spiral TWA Configuration and the Modular Antenna Configuration, when tag was oriented along the X and Y axes.

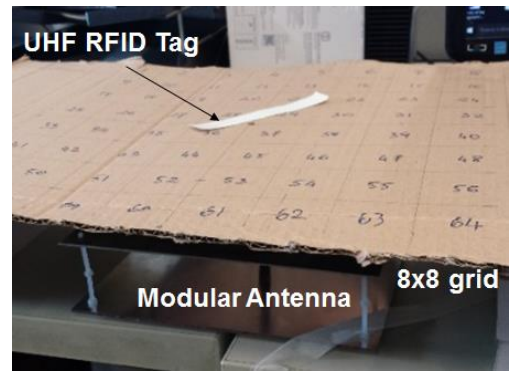

Fig. 11 - System-level measurement setup.

As a result, when the Spiral TWA Configuration is enabled, the generated field is confined close to the antenna surface, and the tag detection is limited up to around $10 \mathrm{~cm}$. By switching to the Modular Antenna Configuration, the reading range extends up to $70 \mathrm{~cm}$. It is worth noting that all the measurements have been performed with $20 \mathrm{dBm}$ reader output power. Thus, the different reading range is only due to the changed field shape generated by the two antenna configurations.

(a)

(b)

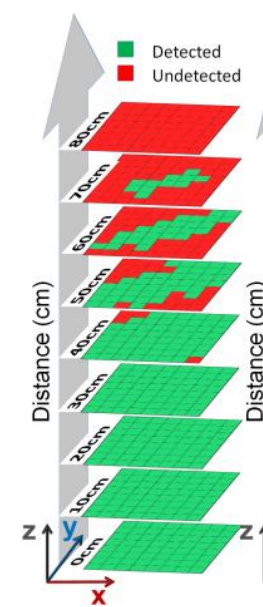

(c)

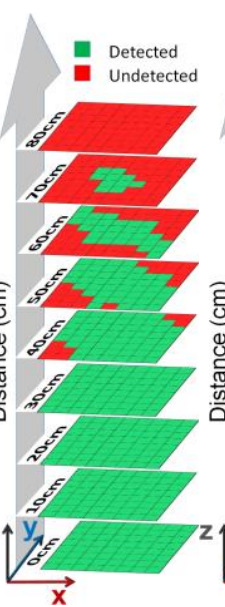

(d)

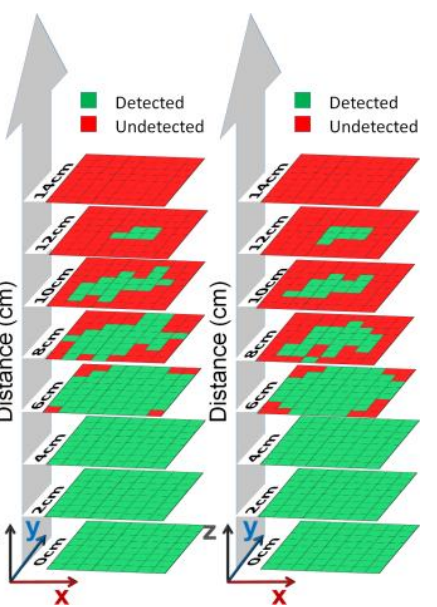

Fig. 12 - Tag detection tests for the Reconfigurable Modular Antenna, by considering a LAB-ID Inlay UH100 tag: (a) X-oriented and (b) Y-oriented tag by enabling the Modular Antenna Configuration; (c) X-oriented and (d) Y-oriented tag by enabling the Spiral TWA Configuration. The tests have been performed by varying the distance and position of the UH100 tag with respect to antenna surface, in an $8 \times 83$-cm-square cells grid (Fig. 11).

To better highlight the different antenna performance when enabling the two operating modes, a further test has been carried out taking into account a stack of eleven UHF RFID tags as described in [32]. Specifically, eleven LAB-ID Inlay UH414 [40] tags have been placed in a stacked configuration at a distance of $2 \mathrm{~cm}$ from each other, up to an overall height of $20 \mathrm{~cm}$. Tags are separated by a foam layer and the lower tag has been placed directly on the case surface. The stacked tags readability has been 
tested on a surface subdivided into $3 \times 3$ cells (cells sizes are about $7 \times 7 \mathrm{~cm}^{2}$ ). The total number of tags detected in a $10 \mathrm{~s}$ time interval has been recorded, when varying the tag orientation and the stack position on the antenna surface. The reader output power has been set to $23 \mathrm{dBm}$. Results are shown in Fig. 13 for both orthogonal tag orientations, and for both the antenna operating modes.

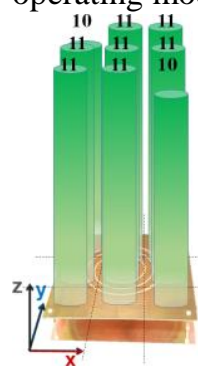

(a)

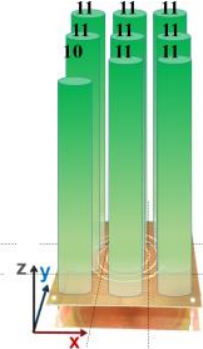

(b)

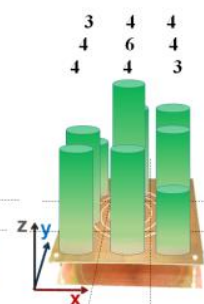

(c)

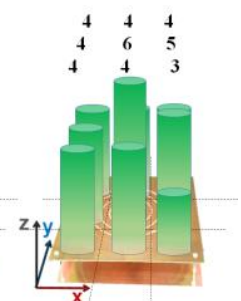

(d)

Fig. 13 - Number of detected tags on the antenna surface by employing eleven LAB-ID UH414 stacked tags at a distance of 2 cm each other, up to a height of 20

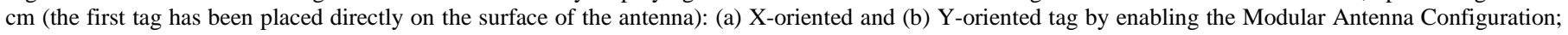
(c) X-oriented and (d) Y-oriented tag, by enabling the Spiral TWA Configuration.

It is apparent that only few tags can be detected when the Spiral TWA Configuration is enabled. As expected, it is possible to read the highest number of stacked tags, i.e. 6 , only when the stack is placed at the antenna center. On the other hand, by switching to the Modular Antenna Configuration, almost all the tags can be easily detected, despite some missing detection when the stack is placed close to the antenna corner.

However, as discussed in [4], a tag detection prediction through numerical simulations would be useful to optimize the radiating element layout, especially when the RFID tag typology is known a priori. In this way, the antenna could be optimized for the specific operating scenario and tag typology.

Thus, in this Section a numerical analysis of the NF UHF RFID radio link is described. Specifically, the tag and reader antennas, and the space between them, have been considered as a linear two-ports network. The numerical evaluation of the entries of the impedance matrix $\underline{\underline{Z}}$ has been performed through the commercial numerical tool CST Microwave Studio ${ }^{\circledR}$.

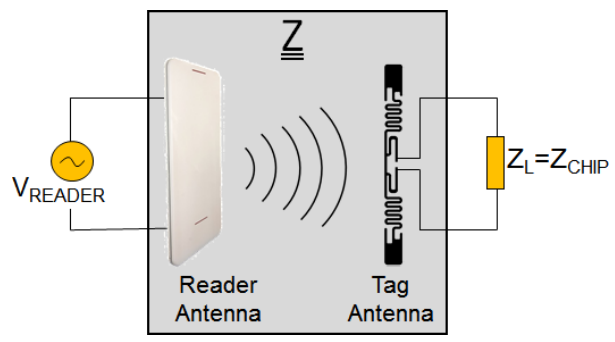

Fig. 14 - Schematic representation of a NF-UHF RFID system consisting in a reader antenna and a tag antenna connected to the RFID chip.

A number of commercial tag layouts have been imported in the numerical model, together with the proposed antenna. Thus, the wireless power transfer efficiency $(P T E)$ between the reader and the tag has been computed to qualitatively estimate the power delivered to the tag chip when varying the tag position. The PTE is defined as the ratio between the power dissipated in the load of the tag antenna (chip), $P_{T}$, and the input power accepted by the reader antenna, $P_{R}$ :

$P T E=\frac{P_{T}}{P_{R}}=\frac{1 / 2 \Re\left\{Z_{L}\right\}\left|I_{2}\right|^{2}}{1 / 2 \Re\left\{Z_{I N}\right\}\left|I_{1}\right|^{2}}=\left|Z_{21}\right|^{2} \frac{\Re\left\{Z_{L}\right\} / \Re\left\{Z_{I N}\right\}}{\left|Z_{L}+Z_{22}\right|^{2}}$

where $Z_{\text {in }}$ is the input impedance of the two-ports network when the latter is connected to $Z_{L}$. As apparent from (1), the PTE expression is given by the square of the mutual impedance amplitude (proportional to the open-circuit voltage at the tag antenna) times a coefficient that includes the tag loading.

The numerical PTE distributions and the measured Received Signal Strength Indicator (RSSI) have been compared. Specifically, to exclude the effect of the RFID chip non-linearity and unpredictable measurement errors, the two parameter distributions have been normalized. For such a reason, and for sake of simplicity, the $\mathrm{Z}_{\mathrm{L}}$ considered for the PTE computation has been chosen equal to the conjugate matching of the simulated antenna input impedance at $866 \mathrm{MHz}$, which represents the typical condition in an operating scenario. To measure the RSSI distributions, the measurement setup shown in Fig. 11 has been used, with a reader output power equal to $20 \mathrm{dBm}$. Thus, the RSSI values were measured and collected for 10 seconds at each cell, and the average value has been computed. The normalized distributions at a distance of $10 \mathrm{~cm}$ from the antenna surface are shown in Fig. 15 when the Spiral TWA Configuration is enabled and when the Inlay UH100 (LAB-ID) tag is oriented toward either the X or the Y axis (Fig. 1). Similarly, in Fig. 16 the same distributions are shown when the Modular Configuration is selected. It is worth noting that the measured RSSI and the numerical PTE distributions are in a good agreement, for both the orthogonal tag 
orientations. As a consequence, from such an analysis the PTE results to be a good candidate to qualitatively predict the RSSI distribution for a given tag typology and reader antenna layout.

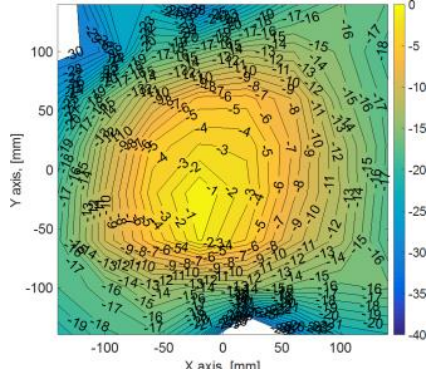

(a)

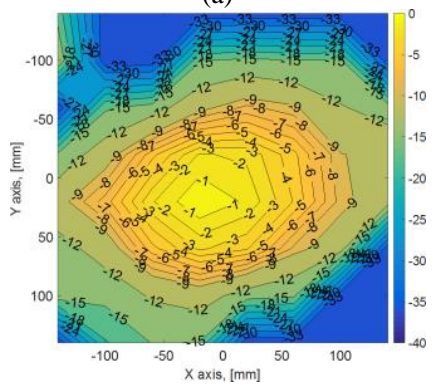

(c)

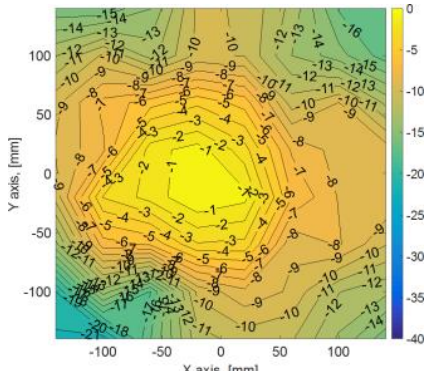

(b)

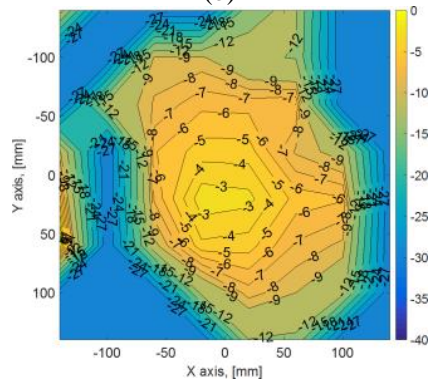

(d)

Fig. 15 - Normalized numerical PTE distribution computed at a distance of $10 \mathrm{~cm}$ from the antenna surface, when the tag is oriented along the (a) X- and (b) Yaxis. Normalized measured RSSI distribution at a distance of $10 \mathrm{~cm}$ from the antenna surface when the LAB-ID Inlay UH100 tag is oriented along the (c) X-and (d) Y- axis. Both results are obtained by considering the antenna operating in the Spiral TWA Configuration.

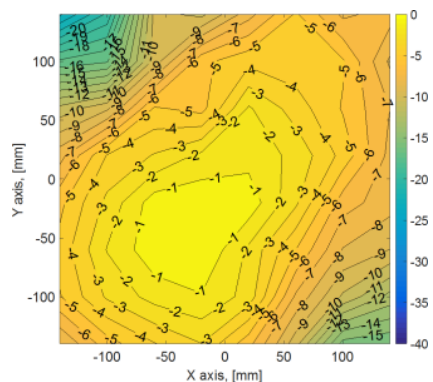

(a)

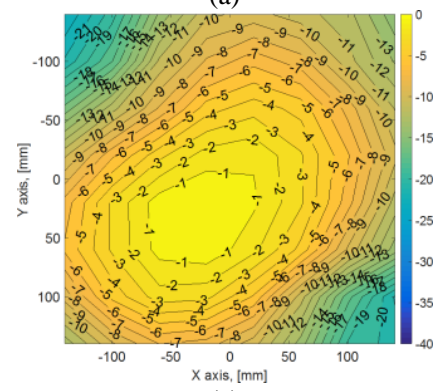

(c)

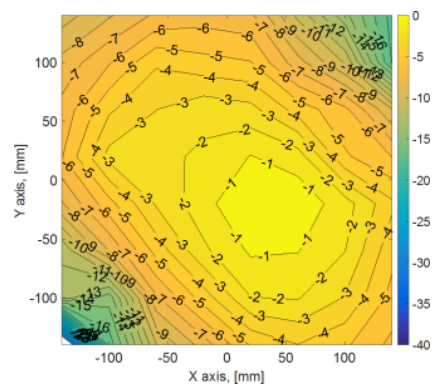

(b)

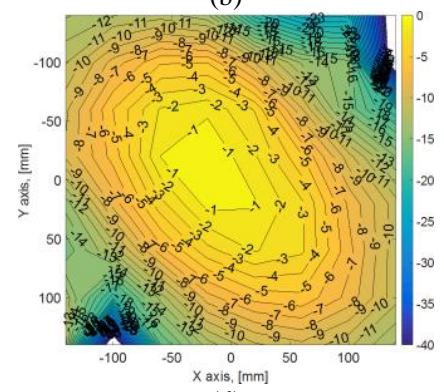

(d)

Fig. 16 - Normalized numerical PTE distribution computed at a distance of $10 \mathrm{~cm}$ from the antenna surface, when the tag is oriented along the (a) X- and (b) Yaxis. Normalized measured RSSI distribution at a distance of $10 \mathrm{~cm}$ from the antenna surface when the LAB-ID Inlay UH100 tag is oriented along the (c) X-and (d) Y- axis. Both results are obtained by considering the antenna operating in the Modular Antenna Configuration.

Moreover, the normalized distributions shown in Fig. 16 are in a good agreement also with the simulated and measured electric field distributions shown in Fig. 10, as expected since a dipole-like RFID tag has been considered.

\section{CONCLUSION}

A Reconfigurable Modular Antenna has been presented for smart point readers employed in near-field applications at the UHF RFID ETSI band (865-868 MHz). It consists of two modules sharing the same aperture: a spiral-shaped travelling wave antenna and an array of four curved resonating slot antennas connected in series to the travelling wave antenna through a printed matching/delay network. By means of an absorptive RF switch, two configurations can be selected (Spiral TWA Configuration 
and Modular Antenna Configuration) on the basis of the particular operating scenario, so changing the field decay rate when moving away from the reader antenna surface. A system level characterization of the reader antenna has also been presented. For an assigned level of the reader output power and independently on the tag orientation, a tag detection up to $14 \mathrm{~cm}$ from the antenna surface is achieved with the Spiral TWA Configuration, while it rises up to $70 \mathrm{~cm}$ if the Modular Antenna Configuration is used.

Finally, a numerical approach based on the PTE calculation has been described to qualitatively predict the RSSI distribution, so demonstrating that the numerical estimations of the electric field distribution and wireless power efficiency are among possible candidates to achieve a tag detection prediction when the tag layout and operating scenario are a priori known, as an alternative to time-consuming measurement campaigns.

\section{ACKNOWLEDGMENT}

This work was supported in part by the Spanish 'Ministerio de Economia y Competitividad' under the project MIRIIEM with ref. TEC-2015-540005-P and by the Gobierno del Principado de Asturias / FEDER under project GRUPIN14-114 .. The authors also acknowledge the support of CST for providing additional resources and technical assistance for the parallel version of CST Microwave Studio ${ }^{\circledR}$.

\section{REFERENCES}

[1] http://www.gs1.org/docs/epc/UHF_Regulations.pdf

[2] F. Fuschini, C. Piersanti, L. Sydanheimo, L. Ukkonen, and G. Falciasecca, "Electromagnetic Analyses of Near Field UHF RFID Systems", IEEE Trans. Antennas Propagation, vol. 58, no. 5, pp. 1759-1770, May 2010.

[3] P.V. Nikitin; K. V. S. Rao; S. Lazar, “An Overview of Near Field UHF RFID”, 2007 IEEE Int. Conf. RFID, pp. 167-174, March 26-28, 2007.

[4] A. Michel, and P. Nepa, "UHF-RFID Desktop Reader Antennas: Performance Analysis in the Near-Field Region", IEEE Antennas and Wireless Propagation Letters, vol. 15, pp. 1430-1433, 2016.

[5] M. Dobkin, S. M. Weigand, and N. Iye, "Segmented Magnetic Antennas for Near-field UHF RFID”, Microwave Journal, 2007

[6] X. Qing, Z. N. Chen, and C. K. Goh, "UHF near-field RFID reader antenna with capacitive couplers,” Electron. Lett., vol. 46, no. 24, pp. 1591-1592, Nov. 2010.

[7] J. Shi, X. Qing, Z. N. Chen, and C. K. Goh, "Electrically Large Dual-Loop Antenna for UHF Near-Field RFID Reader," IEEE Trans. Antennas Propag., vol. 61, no. 3, pp. 1019-1025, Mar. 2013.

[8] A. Sharma, I. J. G. Zuazola, J. C. Batchelor, and A. Perallos, "Dual Purpose Near- and Far-Field UHF RFID Coil Antenna With Non-Uniformly Distributed-Turns," IEEE Antennas Wirel. Propag. Lett., vol. 14, pp. 1342-1345, 2015.

[9] X. Qing, C. K. Goh, and Z. N. Chen, “A Broadband UHF Near-Field RFID Antenna," IEEE Trans. Antennas Propag., vol. 58, no. 12, pp. 3829-3838, December 2010.

[10] J. Shi, X. Qing, and Z. N. Chen, "Electrically Large Zero-Phase-Shift Line Grid-Array UHF Near-Field RFID Reader Antenna," IEEE Trans. Antennas Propag., vol. 62, no. 4, pp. 2201-2208, Apr. 2014.

[11] M. Daiki, E. Perret, and S. Tedjini, "Design of near field UHF RFID reader antenna integrated into clothing," in 2014 IEEE RFID Technology and Applications Conference (RFID-TA), 2014, pp. 261-265.

[12] X.-D. Wei, H.-L. Zhang, and B.-J. Hu, "Novel Broadband Center-Fed UHF Near-Field RFID Reader Antenna," IEEE Antennas Wirel. Propag. Lett., vol. 14, pp. 703-706, 2015.

[13] C. R. Medeiros, J. R. Costa, and C. A. Fernandes, "RFID Smart Shelf With Confined Detection Volume at UHF," IEEE Antennas Wirel. Propag. Lett., vol. 7, pp. 773-776, 2008.

[14] C. R. Medeiros, J. R. Costa, and C. A. Fernandes, "RFID Reader Antennas for Tag Detection in Self-Confined Volumes at UHF," IEEE Antennas Propag. Mag., vol. 53, no. 2, pp. 39-50, Apr. 2011.

[15] C.-Y. Wu, J. Du, A.-K. Ren, and J.-Y. Li, “Large-area RFID reader antenna for smart shelf at UHF,” Electron. Lett., vol. 48, no. 15, pp. 899-900, Jul. 2012.

[16] A. Ren, C. Wu, Y. Gao, and Y. Yuan, “A Robust UHF Near-Field RFID Reader Antenna,” IEEE Trans. Antennas Propag., vol. 60, no. 4, pp. 1690-1697, Apr. 2012.

[17] A. Michel, A. Buffi, R. Caso, P. Nepa, G. Isola, and H. T. Chou, "Design and performance analysis of a planar antenna for near-field UHF-RFID desktop readers," in Microwave Conference Proceedings (APMC), 2012 Asia-Pacific, 2012, pp. 1019-1021.

[18] A. Michel, R. Caso, A. Buffi, P. Nepa, and G. Isola, "Meandered TWAS array for near-field UHF RFID applications," Electron. Lett., vol. 50, no. 1, pp. 17-18, Jan. 2014.

[19] A. Michel, R. Caso, A. Buffi, P. Nepa, and G. Isola, "An array of meander Travelling Wave Antennas for near-field UHF-RFID readers," in 2013 IEEE Antennas and Propagation Society International Symposium (APSURSI), 2013, pp. 1732-1733.

[20] Z. Xing, L. Wang, C. Wu, and K. Wei, "Study of broadband near-field antenna for ultra-highfrequency radio frequency identification applications," IET Microw. Antennas Propag., vol. 5, no. 14, pp. 1661-1669, Nov. 2011.

[21] C. Cho, C. Lee, J. Ryoo, and H. Choo, "Planar Near-Field RFID Reader Antenna for Item-Level Tagging," IEEE Antennas Wirel. Propag. Lett., vol. 10, pp. 1100-1103, 2011.

[22] L. Shen, H. Xiang, W. Tang, W. Zhuang, and J. Ma, “An end-fire dipole array for big interrogation zone of near-field RFID,” in 2015 IEEE International Symposium on Antennas and Propagation USNC/URSI National Radio Science Meeting, 2015, pp. 1582-1583.

[23] J. K. Pakkathillam, M. Kanagasabai, C. Varadhan, and P. Sakthivel, “A Novel Fractal Antenna for UHF Near-Field RFID Readers,” IEEE Antennas Wirel. Propag. Lett., vol. 12, pp. 1141-1144, 2013.

[24] A. S. Andrenko and M. Kai, "Novel design of UHF RFID near-field antenna for smart shelf applications," in Microwave Conference Proceedings (APMC), 2013 Asia-Pacific, 2013, pp. 242-244.

[25] A.S. Andrenko, "Optimized near-field antenna for UHF RFID smart shelf applications," IEEE International Symposium on Antennas and Propagation \& USNC/URSI National Radio Science Meeting, pp.1576-1577, 19-24 July 2015

[26] J. Pakkathillam; M. Kanagasabai; M. Alsath, "A Compact Multiservice UHF RFID Reader Antenna for Near field and Far field Operations," in IEEE Antennas and Wireless Propagation Letters, vol.PP, no.99, pp.1-1 
[27] A. Michel, P. Nepa, X. Qing, and Z. N. Chen, “Antennas for UHF Near-field RFID Readers," to appear on IEEE Antennas and Propag. Magazine

[28] A. Michel, A. Buffi, R. Caso, and P. Nepa, "A two-element modular antenna for near-field UHF RFID applications," 1st URSI Atlantic Radio Science Conference (URSI AT-RASC), 2015, pp.1-1, 16-24 May 2015

[29] A. Michel, A. Buffi, R. Caso, and P. Nepa, "A scalable modular antenna configuration to extend the detection volume of a nearfield UHF-RFID desktop reader," IEEE International Symposium on Antennas and Propagation \& USNC/URSI National Radio Science Meeting, pp.1766-1767, 19-24 July 2015

[30] A. Michel, R. Caso, A. Buffi, P. Nepa, and G. Isola, "Modular antenna for reactive and radiative near-field regions of UHF-RFID desktop readers", URSI-GASS, Beijing, China, August 2014.

[31]R. Caso, A. Michel, A. Buffi, and P. Nepa, " A modular antenna for UHF RFID near-field desktop reader," IEEE RFID Technology and Applications Conference (RFID-TA), Tampere, Finland, 11-13 September 2014.

[32] A. Michel, R. Caso, A. Buffi and P. Nepa, "Multifunctional modular antenna for near-field ultra-high frequency radio frequency identification readers," in IET Microwaves, Antennas \& Propagation, vol. 10, no. 8, pp. 843-849, 2016

[33] T. Yamagajo and M. Kai, "A circulary polarized planar antenna for near field and far filed communication systems," in 2015 IEEE International Symposium on Antennas and Propagation USNC/URSI National Radio Science Meeting, 2015, pp. 1570-1571.

[34] L. Borja, A. Belenguer, J. Cascon, and J. R. Kelly, "A Reconfigurable Passive UHF Reader Loop Antenna for Near-Field and Far-Field RFID Applications," IEEE Antennas Wireless Propagation Letters, vol. 11, pp. 580-583, 2012.

[35]R. Caso, A. Michel, M. R. Pino, and P. Nepa, "Dual-Band UHF-RFID/WLAN Circularly Polarized Antenna for Portable RFID Readers," IEEE Transactions on Antennas and Propagation, vol.62, no.5, pp.2822-2826, May 2014

[36] http://www.analog.com/media/en/technical-documentation/data-sheets/ADG901_902.pdf

[37] A. Arboleya, Y. Álvarez and F. Las-Heras, "Millimeter and submillimeter planar measurement setup," 2013 IEEE Antennas and Propagation Society International Symposium (APSURSI), Orlando, FL, 2013, pp. 1-2

[38] http://www.caenrfid.it/en/CaenProd.jsp?mypage=3\&parent=60\&idmod=836

[39] http://www.lab-id.com/datasheet/inlay_UHF/UH100.pdf

[40]http://www.lab-id.com/datasheet/inlay_UHF/UH414.pdf

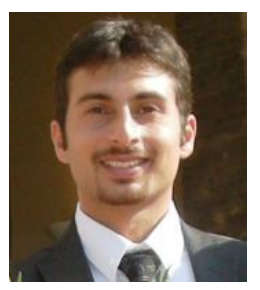

Andrea Michel received the B.E., M.E. (summa cum laude) and $\mathrm{PhD}$ degrees in telecommunications engineering from the University of Pisa, Pisa, Italy, in 2009, 2011 and 2015, respectively. Since 2015 he is a PostDoc researcher in applied electromagnetism at the Microwave\&Radiation Laboratory, Dept. of Information Engineering, University of Pisa. His research topics focus on design of integrated antenna for communication systems and smart antennas for near field UHF-RFID readers. In 2014 he was a Visiting Scholar at the ElectroScience Laboratory, The Ohio State University, Columbus, OH, USA. During this period, he has been involved in research on a theoretical analysis on the accuracy of a novel technique for deep tissue imaging. Recently, he is working on the design of antennas for automotive applications, MIMO systems and wearable communication systems, in collaboration with other research institutes and companies. Dr. Michel was a recipient of the Young Scientist Award from the International Union of Radio Science, Commission B, in 2014, 2015 and 2016. In 2016, he received the Best Paper Honorary Mention from the IEEE International Conference on RFID Technology and Applications, Shunde, Guangdong, China

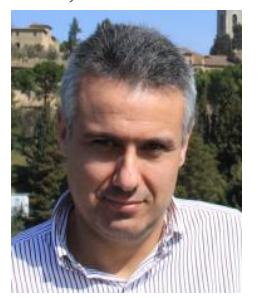

Marcos R. Pino was born in Vigo, Spain, in 1972. He received the M.Sc. and Ph.D. degrees in telecommunication engineering from the University of Vigo, Spain, in 1997 and 2000, respectively. In 1998, he was a Visiting Scholar at the ElectroScience Laboratory, The Ohio-State University, Columbus, OH, USA. From 2000 to 2001, he was with the University of Vigo as Assistant Professor. Since 2001, he has been with the Electrical Engineering Department, University of Oviedo, Spain, where he is currently Associate Professor teaching courses on communication systems and antenna design. His research interests include antenna design for different applications (RFID, satellite broadcasting, ...), both near-field and far-field measurement techniques and facilities, efficient computational techniques applied to solve EM problems, such as evaluation of radar cross section or EM scattering from targets in the presence of rough surfaces.

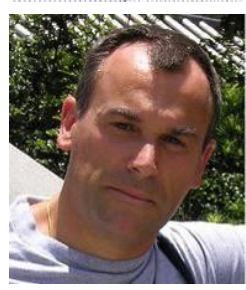

Paolo Nepa received the Laurea (Doctor) degree in electronics engineering (summa cum laude) from the University of Pisa, Italy, in 1990. Since 1990, he has been with the Department of Information Engineering, University of Pisa, where he is currently an Associate Professor. In 1998, he was at the Electro Science Laboratory (ESL), The Ohio State University (OSU), Columbus, OH, as a Visiting Scholar supported by a grant of the Italian National Research Council. At the ESL, he was involved in research on efficient hybrid techniques for the analysis of large antenna arrays. His research interests include the extension of high-frequency techniques to electromagnetic scattering from material structures and its application to the development of radio propagation models for indoor and outdoor scenarios of wireless communication systems. He is also involved in the design of wideband and multiband antennas, mainly for base stations and mobile terminals of communication systems, as well as in the design of antennas optimized for near-field coupling and focusing. More recently he is working on channel characterization, wearable antenna design and diversity scheme implementation, for body-centric communication systems. In the context of RFID systems, he is working on techniques and algorithms for UHF-tag localization and RFID-based smart shelves. He is an Associate Editor of the IEEE Antennas and Wireless Propagation Letters. Dr. Nepa received the Young Scientist Award from the International Union of Radio Science, Commission B, in 1998. 